\title{
A concern over terminology in vaccine effectiveness studies
}

Benjamin J Cowling ${ }^{1}$, Sheena G Sullivan ${ }^{2,3,4}$

1. WHO Collaborating Centre for Infectious Disease Epidemiology and Control, School of Public Health, The University of Hong Kong, Hong Kong Special Administrative Region, China

2. WHO Collaborating Centre for Reference and Research on Influenza at the Peter Doherty Institute for Infection and Immunity, Melbourne, Victoria, Australia

3. Department of Epidemiology, Fielding School of Public Health, University of California, Los Angeles, Los Angeles, California

4. Centre for Epidemiology and Biostatistics, Melbourne School of Population and Global Health, University of Melbourne, Melbourne, Victoria, Australia

Correspondence: Benjamin J Cowling (bcowling@hku.hk)

Cowling Benjamin J, Sullivan Sheena G. A concern over terminology in vaccine effectiveness studies. Euro Surveill. 2018;23(10):pii=18-00103. https://doi. org/10.2807/1560-7917.ES.2018.23.10.18-00103

Article submitted on 07 Mar 2018 / accepted on 08 Mar 2018 / published on 08 Mar 2018

To the editor: Ongoing systematic monitoring of vaccine effectiveness (VE) provides evidence to support vaccination programmes and policies. A series of recent articles in Eurosurveillance [1-4] and elsewhere [5], continue to provide timely estimates of influenza VE from around the world. These reports are useful for supporting public health decision making on the use of influenza vaccines, which are the best means currently available for reducing the considerable burden of influenza.

However, as we pointed out already, we are concerned that the continued use of the terms 'crude VE' and 'adjusted VE' in many such papers is unhelpful [6]. The term vaccine effectiveness implies an attempt to measure a causal estimate, i.e. the effect of vaccination on the risk of an infection-related outcome such as medically-attended influenza or hospitalisation, and not merely the association of vaccination and (absence of) influenza virus infection [6]. The term 'effect' should consequently be reserved for the reporting of unbiased estimates of a causal effect, or at least the reasonable attempt to generate such an unbiased estimate.

Epidemiologists have long been cautioned against drawing causal inferences from observational studies [7]. Indeed, some specialist epidemiology journals discourage use of the word 'effect'. We are instead encouraged to comment on whether a particular factor is 'associated with reduced risk of...' rather than stating definitively that it 'reduced the risk of...' [8]. However it is increasingly realised that observational studies can, in certain cases, permit inferences on cause and effect relationships $[9,10]$.

In a test-negative design study of influenza vaccine effectiveness against medically-attended influenza, a crude association between case vs control status and influenza vaccination history may not reflect the true strength of a causal effect. The association may be confounded by a factor such as age, i.e. a factor that has a causal effect on both the exposure (vaccination) and the outcome (influenza virus infection). An estimate of the effect of vaccination on risk of medicallyattended influenza would need to take into account any potential confounding by age or other factors, which may be achieved by methods such as stratification or regression analysis. Typically, the VE estimate would be derived from the antilog of the estimated coefficient for vaccination in a regression model that included potential confounders; this value is often referred to as the adjusted odds ratio (AOR). In the special case where all potential confounders, but no other variables, are included as covariates in the regression model, and in the absence of other biases [6], it is possible to interpret the AOR as an estimate of a causal effect, and estimate the VE as one minus the AOR.

In contrast, crude (i.e. unadjusted) estimates are unlikely to be an accurate estimate of the VE, because of confounding. Discussion of crude associations should therefore remain on the odds ratio scale to prevent the reader assuming they are a measure of effect. The causal effect is not the quantity that has been adjusted for confounding, it is based on an estimate from an analysis that accounts for confounding. We therefore recommend avoiding the terms 'crude VE' and 'adjusted VE'. In summary tables, it is unnecessary to report unadjusted odds ratios or 'crude VE'. If authors wish to compare unadjusted and adjusted odds ratios they could be presented separately, for example in an appendix. 
Benjamin J Cowling has received research funding from Sanofi. Sheena G Sullivan has no conflict of interest to declare.

\section{Authors' contributions}

Conception: BJC and SGS. Wrote first draft: BJC. Edited text and approved final version: BJC and SGS.

\section{References}

1. Skowronski DM, Chambers C, De Serres G, Dickinson JA, Winter AL, Hickman R, et al. Early season co-circulation of influenza $A\left(\mathrm{H}_{3} \mathrm{~N}_{2}\right)$ and $B($ Yamagata): interim estimates of $2017 / 18$ vaccine effectiveness, Canada, January 2018. Euro Surveill. 2018;23(5):18-00035. https://doi.org/10.2807/15607917.ES.2018.23.5.18-00035 PMID: 29409570

2. Castilla J, Navascués A, Casado I, Pérez-García A, Aguinaga A, Ezpeleta G, et al. Interim effectiveness of trivalent influenza vaccine in a season dominated by lineage mismatched influenza B, northern Spain, 2017/18. Euro Surveill. 2018;23(7):18-00057. https://doi.org/10.2807/1560-7917. ES.2018.23.7.18-00057 PMID: 29471624

3. Chiu SS, Kwan MYW, Feng S, Wong JSC, Leung CW, Chan ELY, et al. Interim estimate of influenza vaccine effectiveness in hospitalised children, Hong Kong, 2017/18. Euro Surveill. 2018;23(8):18-00062. https://doi.org/10.2807/1560-7917. ES.2018.23.8.18-00062 PMID: 29486830

4. Rondy M, Kissling E, Emborg HD, Gherasim A, Pebody R, Trebbien R, et al. I-Move/I-Move Group. Interim 2017/18 influenza seasonal vaccine effectiveness: combined results from five European studies. Euro Surveill. 2018;23(9):18ooo86. https://doi.org/10.2807/1560-7917.ES.2018.23.9.1800086 PMID: 29510782

5. Flannery B, Chung JR, Belongia EA, McLean HQ, Gaglani M, Murthy K, et al. Interim Estimates of 2017-18 Seasonal Influenza Vaccine Effectiveness - United States, February 2018. MMWR Morb Mortal Wkly Rep. 2018;67(6):180-5. https://doi. org/10.15585/mmwr.mm6706a2 PMID: 29447141

6. Sullivan SG, Cowling BJ. 'Crude vaccine effectiveness' is a misleading term in test-negative studies of influenza vaccine effectiveness. Epidemiology. 2015;26(5):e6o. https://doi. org/10.1097/EDE.0000000000000343 PMID: 26133018

7. Petitti DB. Associations are not effects. Am J Epidemiol. 1991;133(2):101-2. https://doi.org/10.1093/oxfordjournals.aje. a115848 PMID: 1985440

8. Heart Group Editors. Statement on matching language to the type of evidence used in describing outcomes data. Heart. 2013;99(18):e1. https://doi.org/10.1136/heartjnl-2012-303287 PMID: 23175130

9. Maldonado G, Greenland S. Estimating causal effects. Int J Epidemiol. 2002;31(2):422-9. https://doi.org/10.1093/ intjepid/31.2.422 PMID: 11980807

10. Vandenbroucke JP, Broadbent A, Pearce N. Causality and causal inference in epidemiology: the need for a pluralistic approach. Int J Epidemiol. 2016;45(6):1776-86. https://doi.org/10.1093/ ije/dyv341 PMID: 26800751

\section{License and copyright}

This is an open-access article distributed under the terms of the Creative Commons Attribution (CC BY 4.0) Licence. You may share and adapt the material, but must give appropriate credit to the source, provide a link to the licence, and indicate if changes were made.

This article is copyright of the authors, 2018. 